\title{
Design Optimization of Machine Accessories through Analysis of their Elements Deformation in NX NASTRAN
}

\author{
M. V. Nekhoroshev \\ Institute of Engine and Power Plant Engineering \\ Samara University \\ Samara, Russia \\ maxnogood@ssau.ru \\ K. A. Bezsonov \\ Institute of Engine and Power Plant Engineering \\ Samara University \\ Samara, Russia \\ kirillbez2306@gmail.com
}

\author{
A. V. Meshcheryakov \\ Institute of Engine and Power Plant Engineering \\ Samara University \\ Samara, Russia \\ al-mes1@mail.ru \\ A. P. Shulepov \\ Institute of Engine and Power Plant Engineering \\ Samara University \\ Samara, Russia \\ shulepov_SI@mail.ru
}

\begin{abstract}
The article presents design optimization of machine accessories used for milling, according to required accuracy of geometrical parameter of machined part. The authors propose a computerized engineering analysis of deformation of device elements when cutting forces are applied. NX Nastran software is used for analysis. A parameter-oriented 3D model of a device was created for the procedure. The model was built with NX CAM software using the variable parameterization method that provides for developing multiplechoice flexible models of device assembly. The authors analyzed multiple designs of device elements which, when deformed under cutting forces, directly influenced the accuracy of geometrical dimension acquired after processing. It is shown that application of NX Nastran software and a parameter-oriented 3D model of the device facilitates the process of device redesign according to technical requirements for operation and machining conditions, thus significantly reducing engineering time and level of errors that occur throughout design and production stages.
\end{abstract}

Keywords- machine accessories; parameter-oriented model; deformation; analysis

\section{Introduction}

Today every enterprise uses production accessories, which mostly consist of machine accessories. Workplaces should be timely equipped with such devices to ensure adequate result and quality of production. The time required for engineering and manufacturing of machine accessories could be reduced through application of CAD/CAM systems. Building parameter-oriented 3D models and databases for designs applied in device production is one of the most important functions of such systems. It provides new versions of object design by changing only a limited number of its parameters [1].
Design of machine accessories should satisfy the defined conditions of production and operation and be chosen with due regard to various factors influencing the device [2]. Factors defined by unique operational conditions of accessories fall into the following categories: structural, which depends on dimensions, configuration, and accuracy of working; technological, which includes a machining method, type of movement (shift) of a work piece, type of machine, features of tools, and working modes; practical, which is associated with distinctive features of a manufacturing process, type of manufacture, and flexibility when it comes to changing working objects; operational, which is defined by required calibration stability during operation and maintenance ability of a device.

Calculations for accuracy are made during device design stage. These calculations do not consider possible deformations of blanks and device elements caused by applied cutting forces during working. Engineering analysis using parameter-oriented 3D models is performed to estimate status of a device and a blank resulting from power and other type factors in CAE systems [3, 4].

In the study [5], the authors consider a method of variation parameterization, which is based on sketching with various parametric links superimposed on the sketch objects and userimposed constraints in the form of an equation system that determines the parametric dependencies. This method of parameterization enables development of multi-variant and flexible fixture assembly model, as all the modeling operations are hierarchically linked in the design tree, providing for further structural optimization of a device depending on operational conditions.

In article [6], the authors propose a new approach to inclusion of tolerances on 3D models by determining 
configurations with positional, orientation and form defects. This approach provides an assembly result closer to the real assembly of the mechanical system. Integrating tolerances enables the simulation and visualization of the mechanical assemblies' behavior in their real configurations.

In [7] the authors analyze the usage of simulated data as offset data in the machining process on $\mathrm{CNC}$ equipment. They also consider design of jigs and fixtures, which ensure a geometrically-accurate final product.

A new modeling mechanism known as CADWE (Computer-Aided Design-While-Engineering), which combines CAD and CAE software, is proposed in paper [8]. This approach provides a single modeling mechanism with a coordinated structure of data and a top-down distribution of synchronous changes in assembly design.

The authors of [9] study geometric modeling of spatial constraints of parts and assemblies. For each type of position and shape tolerances, the paper describes a method for building a configuration space, a point wherein helps obtain various positions of the marked frame of this surface within the tolerance; i.e. a full description of the $3 \mathrm{D}$ position of this surface.

Optimized design could be achieved through implementation of efficiency functions that include changes in design variables, such as dimensions of parts, material properties and so on. The focus of optimization is efficiency functions considered as capacity limitations, which generally arise when solving engineering problems $[10,11]$. Computing CAD models in CAE system helps optimize the materials used in parts' design $[12,13]$.

The authors of [14] consider the influence of working load when calculating the deflection occurred in assemblies. Both manufacturing error and deformation error are taken into consideration to establish deviation calculation model based on Jacobian-Torsor model. A tailstock is taken as an example to verify the feasibility of the proposed method.

It may be possible to use design data and estimations to evaluate dimensions change during the assembly. For example, a model for predicting the dimension, designed tolerances, designed assembly processes, and measurement data is used for revising the assembly sequence [15].

Therefore, having analyzed the sphere of interest we conclude that persistent efforts are undertaken both in application of various methods of building parameter-oriented 3D models of machine accessories, and in development of CAE systems for operation analysis and optimization of device designs.

\section{Materials and Methods}

The present article presents design optimization of machine accessories according to required accuracy of a part's geometrical parameter. The device is intended for slot milling of a bellcrank, (see Figure 1 for a 3D model).

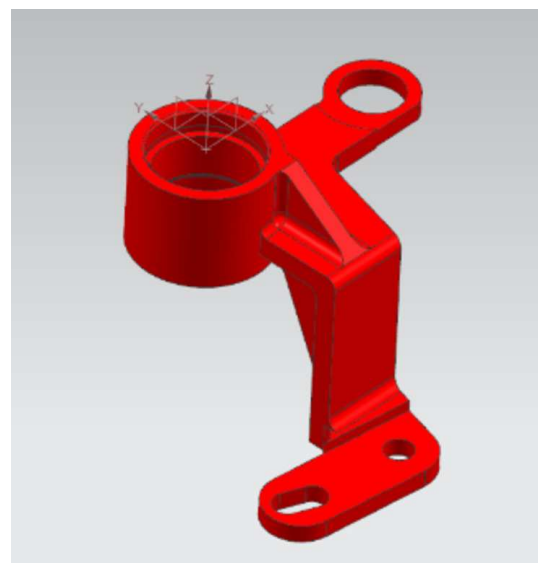

Fig. 1. Bellcrank 3D Model

When treating the part, resulting dimension A between reference hole axis and slot axis should be $60 \mathrm{~mm}$ with tolerance $\mathrm{T}= \pm 0.05 \mathrm{~mm}$ (Fig. 2). A 3D model of the device with installed blank is given in Figure 3 . The models of a blank and device were built with NX CAD software using variable parameterization method that provides for developing multiple-choice flexible models of device assembly. When applying this method, the design process could be easily split into a required number of interconnected stages (for instance, sketch, detailed design, etc.). This could significantly reduce the number of errors by virtue of using physical links between the parts, and provide for quick change of dimensions and structural elements of the general assembly. A cylinder peg was used as a supporting bearing in the design to neutralize possible deformation when treating a blank.

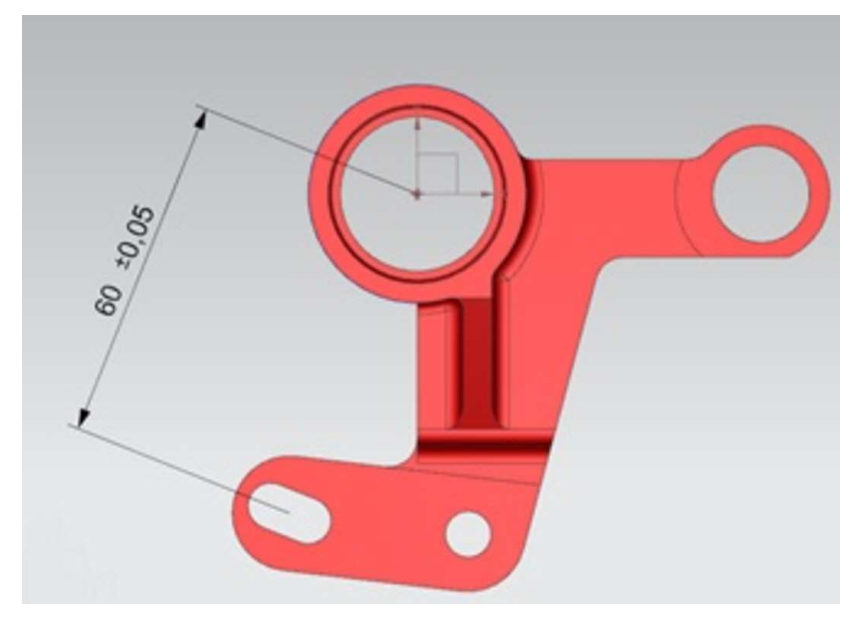

Fig. 2. Required Dimension after Treatment 


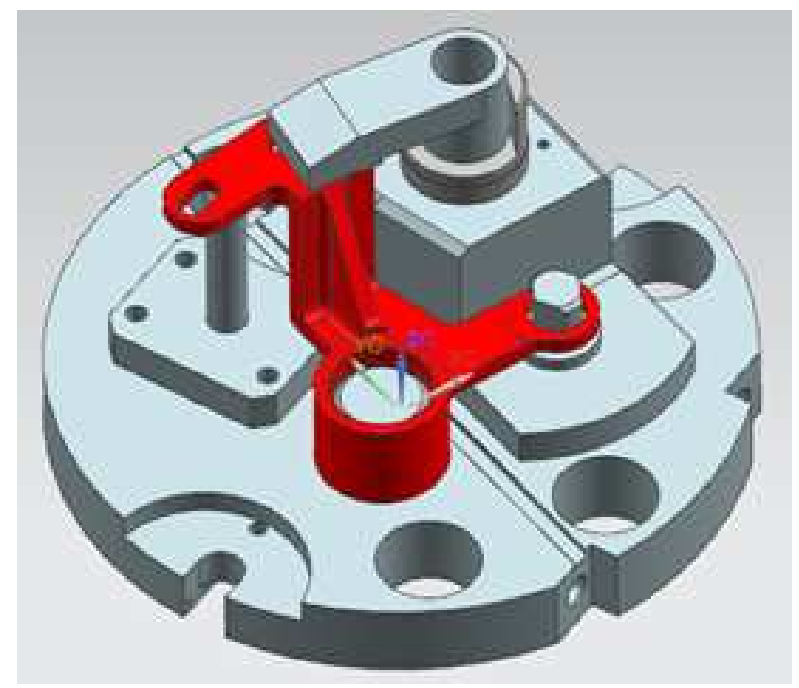

Fig. 3. Device 3D Model

Deformation of assembly elements resulting from applied cutting forces is analyzed in the environment of NX Nastran of Siemens NX software. CAE system NX Nastran by Siemens PLM Software is used for computerized engineering analysis of designed articles employing the finite element method. Combined with additional solvers, this software calculates both static and dynamic linear and nonlinear problems of engineering analysis.

In the course of analysis, the authors took into consideration strain occurring in the assembly elements and possible shifts of these elements, which influence the resulting specified operational dimension. This way, it is possible to evaluate operating capacity of a device under the actual load of cutting forces and resulting slot misalignment after the load is removed.

\section{Results and Discussion}

Deformation of assembly elements (device-blank) resulting from applied cutting forces is analyzed in stages.

During the first stage, device assembly (Fig. 3) received maximum possible load of cutting forces applied during this type of working. The occurring strains and shifts in grid nodes of finite elements (deformation) were analyzed. Estimation has shown that the maximum value of strain of elements/nodes was $409.5 \mathrm{MPa}$, which does not exceed the limit of a yield point of the analyzed material that equals $950 \mathrm{MPa}$ (Fig. 4). At the same time, the maximum value of shift in nodes was $0.089 \mathrm{~mm}$ (Fig. 5). Once the load is removed, these shifts could result in errors in dimension A exceeding the specified tolerance $\mathrm{T}=0.05 \mathrm{~mm}$ and lead to defective production.

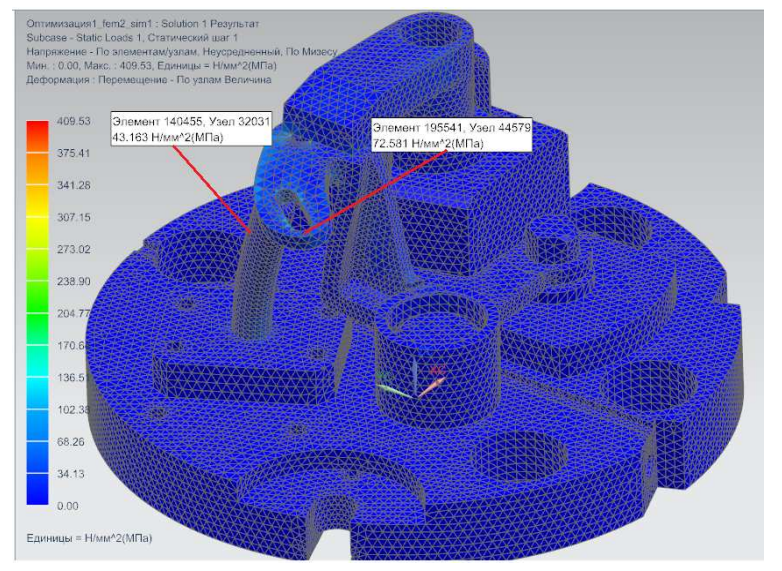

Fig. 4. Total Strain (first version)

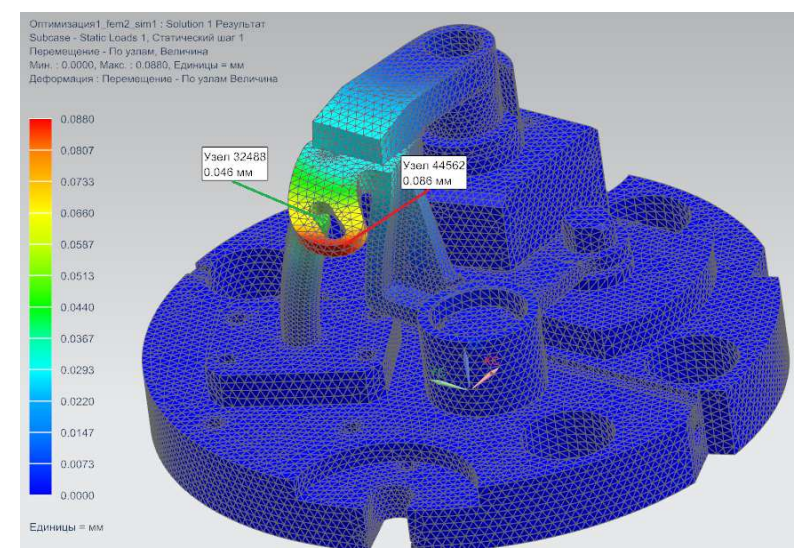

Fig. 5. Total Shift (first version)

Analysis of the resulting shifts in assembly elements leads to conclusion that slot misalignment in the blank is caused by deformation of the blank itself and the support due to its fragility. It should be mentioned that the support has a globe head, so its contact area with the blank is very small (point contact). Moreover, deformation of the support during milling will cause vibrations, thus exacerbating working errors and adversely affecting quality of the treated surface (higher unevenness, form error, etc.).

During the second stage, support design was changed. The part was made more rigid; contact area of support and blank was extended (Fig. 6). This assembly could be produced through casting with further mechanical treatment. 


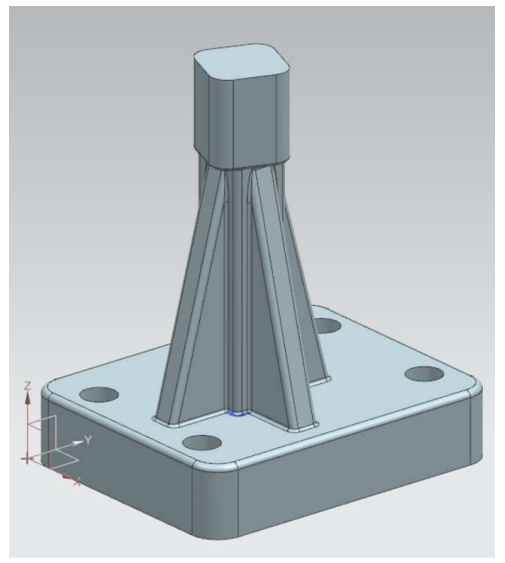

Fig. 6. Support

Estimations of the revised design of the support have shown that maximum value of strain of elements/nodes was $97.48 \mathrm{MPa}$, which is significantly lower than in the first version of the device (Fig. 7).

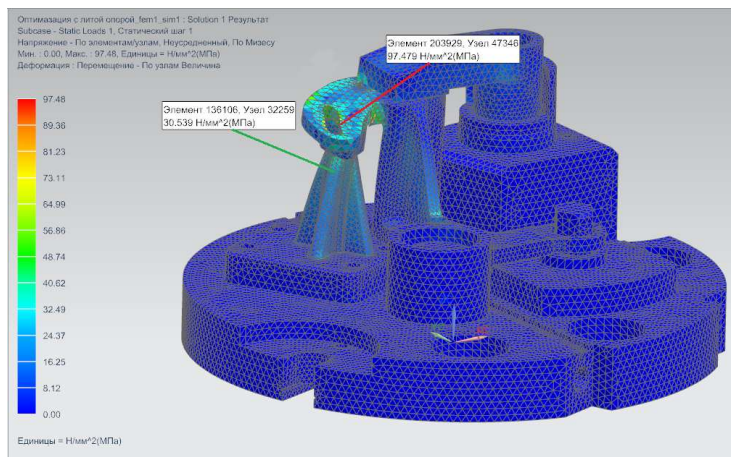

Fig. 7. Total Strain (second version)

The maximum value of shift in nodes was $0.058 \mathrm{~mm}$ (Fig. 8). Redesigned support bore the load much better, but still caused defective production. As in the first case, the deformations exceed the dimension tolerance.

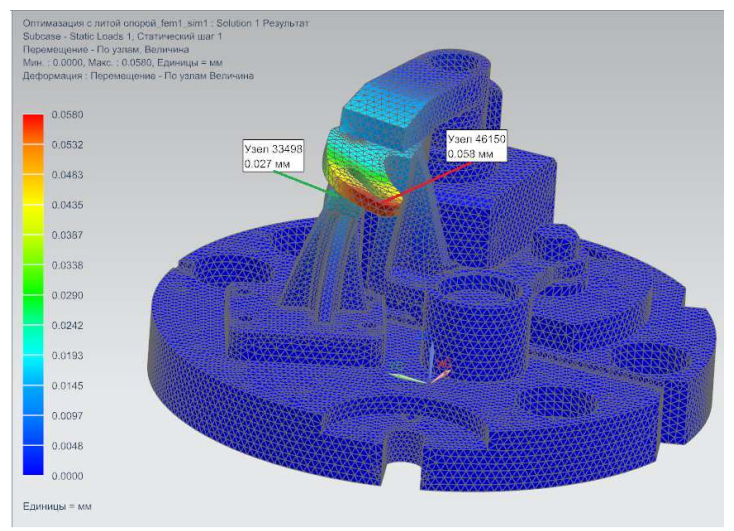

Fig. 8. Total Shift (second version)

During the third stage, a different design of the support was analyzed (Fig. 9). Due to increased dimensions, this assembly is even more rigid and has a greater contact area with the blank.

Moreover, to enhance the device, a setup element was introduced into the support design. This way tooling setup could be performed for operation and then the device could be used not only in $\mathrm{CNC}$ equipment, but also in universal milling machines.

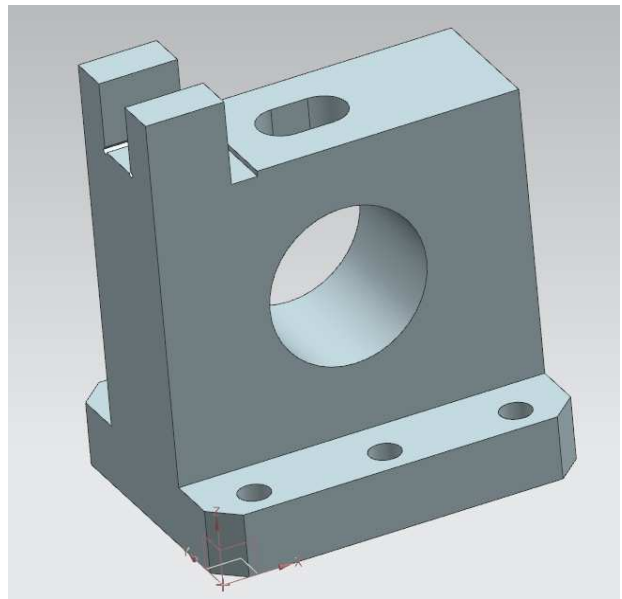

Fig. 9. Support

Calculations made when the proposed support design was used have given the following results. Maximum values of strain were reduced to $20.4 \mathrm{MPa}$ (Fig. 10), which is remarkably lower than those acquired with the first two designs of the support. The maximum value of deformations (shift in nodes) was $0.003 \mathrm{~mm}$ (Fig. 11). This deformation of elements is massively smaller than operational dimension tolerance almost eliminating occurrence of defects during machining.

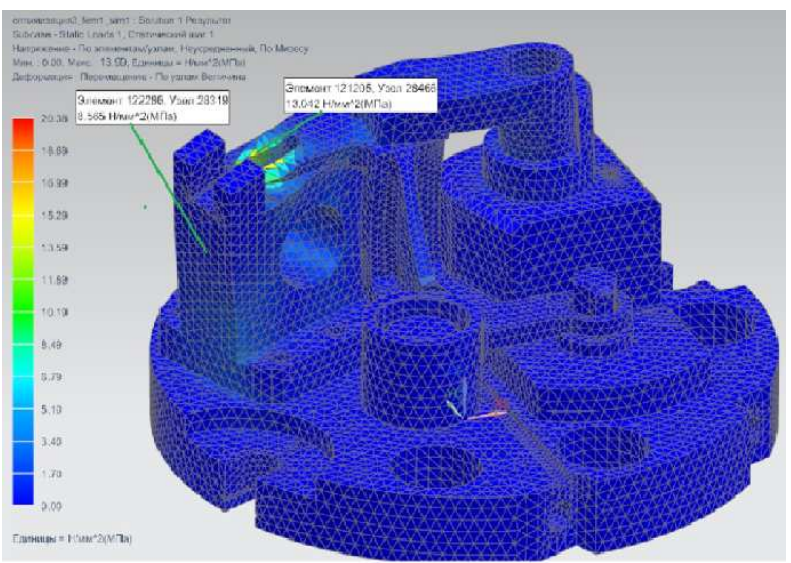

Fig. 10. Total Strain (third version)

The maximum value of deformations (shift in nodes) was $0.003 \mathrm{~mm}$ (Fig. 11). This deformation of elements is massively smaller than operational dimension tolerance. 


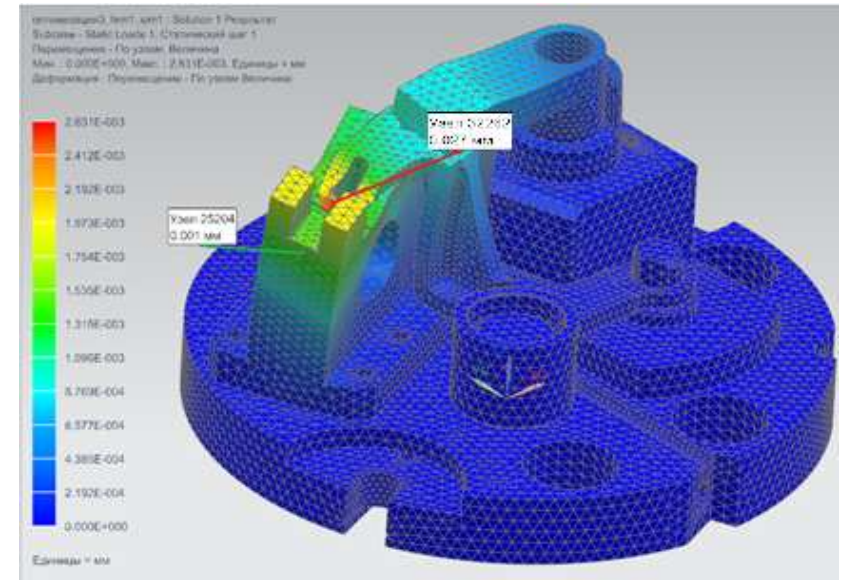

Fig. 11. Total Shift (third version)

As a result, the third version of support design reduces deformations of device elements when cutting force is applied and eliminates defects during slot milling. It is also important that the proposed support design allows for rigidity adjustment by changing diameter of a hole in the support. This way, should the machining conditions change (for example, if the cutting force augments) support features could be adjusted according to new technical requirements for operation through parameterization of the entire assembly.

The suggested method of analysis of machine accessories operation significantly reduces design time of production accessories. It is made possible by application of the parameter-oriented 3D model of the device and its engineering analysis in the CAE system, which grants an opportunity to choose a design that fully satisfies technical conditions of the technological procedure.

\section{Conclusion}

This study includes research and information analysis of publications on parametric modeling of machine accessories combined with engineering analysis of their operation in CAE systems, which proved the relevance of line of research. A method of analysis of device elements' deformation under cutting forces was evaluated in NX Nastran system, which provides for optimization of device design based on the specified operational dimension tolerance during slot milling. Application of NX Nastran software and with NX CAM for computerized engineering analysis facilitates the process of device redesign according to technical requirements for operation and machining conditions, thus significantly reducing design time and level of errors that occur throughout design and production stages.

CAE system is proposed for future integration into CAM model of a device. This approach could be applied for optimization of separate elements of design or for improvement of a device in general based not only on accuracy of geometrical parameter, but also on economic efficiency of the applied device.

\section{References}

[1] P. S. Goncharov, M. Yu. Yeltsov, S. B. Korshikov et al., NX for mechanical engineering designer, Moscow: DMK Press, 2010, pp. 504550 .

[2] A. Gameros, S. Lowth, A. Dragos, A. Nagy-Sochacki, O. Craig, H. R. Siller, "State-of-the-art in fixture systems for the manufacture and assembly of rigid components: A review", International Journal of Machine Tools and Manufacture, pp. 15-30, 2017.

[3] A. I. Ermakov, A. V. Urlapkin, "The influence of a blade Vibrations connectivity on a degree of disturbance of turbine wheels rotation symmetry", Res. J. Appl. Sci,vol. 9, no. 11, pp. 800-805, 2014.

[4] A. I. Ryazanov,"Mathematical model and numerical solution of the process of heating and melting of a traveling cylinder fed into a rocket chamber", ARPN J. Eng. Appl. Sci., vol. 9, pp. 1859-1865, 2014.

[5] A. V. Balaykin, K. A. Bezsonov, M. V. Nekhoroshev, A. P. Shulepov, "Developing Parametric Models for the Assembly of Machine Fixtures for Virtual Multiaxial CNC Machining Centers", IOP Conference Series: Materials Science and Engineering, Volume 302, Issue 1, pp 325-330, 2017.

[6] I. Jbira, M. Tlij, B. Louhichi, A. Tahan "CAD/Tolerancing integration: Mechanical assembly with form defects" Advances in Engineering Software Volume 114, pp. 312-324, December 2017.

[7] A. Hansel, K. Yamazaki, K. Konishi, "Improving CNC machine tool geometric precision using manufacturing process analysis techniques" Procedia CIRP 14, pp. 263-268, 2014.

[8] Z. Pan, X. Wang, R. Teng, X. Cao, "Computer aided design while engineering technology in top down modeling of mechanical product", Comput. Ind. 75, pp. 151-161, 2016.

[9] M. A. Gaer, D. A. Zhuravlev, O. V. Yatsenko "Configuration spaces of parts and assembly surfaces", Bulletin of the Irkutsk state technical university vol. 10 (57) pp. 32-36, 2011.

[10] K.-H. Chang, Product Design Modeling Using Cad/Cae, Academic Press, Kidlington, Oxford, UK, 2014, pp. 25-43.

[11] Y. Bodein, B. Rose, E. Caillaud, "Explicit Reference Modeling Methodology in Parametric CAD System" Computers in Industry 65 (1), pp. 136-147, 2014.

[12] A. V. Balaykin, E. A. Nosova, N.V. Galkina, "Study of the $\mathrm{Ti}-5 \mathrm{Al}-5 \mathrm{Mo}-5 \mathrm{~V}-1 \mathrm{Cr}-1 \mathrm{Fe}$ titanium alloy grain structure uniformity after bending and annealing", IOP Conference Series: Materials Science and Engineering, Vol. 177, I. 1., pp. 140-151, 2017.

[13] V. P. Alexeev, A. V. Balyakin, A. I. Khaimovich, "Influence of the direction of selective laser sintering on machinability of parts from 316 L steel." IOP Conference Series: Materials Science and Engineering, Vol. 177, I.1., pp. 240-264, 2017.

[14] T. Liu, Y. L. Cao, J. Wang, J. X. Yang, "Assembly Error Calculation with Consideration of Part Deformation.”, Procedia CIRP 2016, Vol. 43, pp. 58-63, 2016.

[15] P. Vichare, O. Martin, J. Jamshidi, "Dimensional management for aerospace assemblies: framework implementation with case-based scenarios for simulation and measurement of in-process assembly variations.”, Int. J. Adv. Manuf. Technol. 70 (1-4), pp. 215-230, 2014. 